\title{
Reliability-Aware Design of Spike-Event Neuromorphic Circuits
}

This paper was downloaded from TechRxiv (https://www.techrxiv.org).

\section{LICENSE}

CC BY 4.0

SUBMISSION DATE / POSTED DATE

$10-08-2021 / 12-08-2021$

CITATION

Shaik, Jani Babu; Picardo, Siona Menezes; Singhal, Sonal; Goel, Nilesh (2021): Reliability-Aware Design of Spike-Event Neuromorphic Circuits. TechRxiv. Preprint. https://doi.org/10.36227/techrxiv.15141537.v1

DOI

10.36227/techrxiv.15141537.v1 


\title{
Reliability-Aware Design of Spike-Event Neuromorphic Circuits
}

\author{
Jani Babu Shaik*, Student Member, IEEE, Siona Menezes Picardo, Student Member, IEEE, Sonal \\ Singhal, Member, IEEE and Nilesh Goel, Member, IEEE
}

\begin{abstract}
Very Large Scale Integration (VLSI) based neuromorphic circuits also known as Silicon Neurons (SiNs) emulate the electrophysiological behavior of biological neurons. With the advancement in technology, neuromorphic systems also lead to various reliability issues and hence making their study important. Bias Temperature Instability (BTI) and Hot Carrier Injection (HCI) are the two major reliability issues present in VLSI circuits. In this work, we have investigated the combined effect of BTI and HCI on the two types of integrate-and-fire based SiNs namely (a) Axon-Hillock and (b) Simplified Leaky integrateand-fire circuits using their key performance parameters. Novel reliability-aware AH and SLIF circuits are proposed to mitigate the reliability issues. Proposed reliability-aware designs show negligible deviation in performance parameters after aging. The time-zero process variability analysis is also carried out for proposed reliability-aware SiNs. The power consumption of existing and proposed reliability-aware neuron circuits is analyzed and compared.
\end{abstract}

Index Terms-Neuromorphic circuits, Circuit reliability, BTI, HCI, Integrate-and-fire neuron model.

\section{INTRODUCTION}

$\mathrm{N}$ euromorphic computing primarily aims at emulating electro-physiological behaviours and computations of real biological neurons and neuronal systems using, mixedmode analog/digital VLSI system and was coined by Carver Mead [1]. The concept of neuromorphic computing adopts various computational models based on biological neural systems to develop massively parallel, hybrid processing electronic systems on silicon chips [1]. Progressive microelectronics has been engineered to be highly competent towards processing signals in low-power circuit designs; making them particularly relevant in realizing cognitive functions in the nervous system such as object recognition, data, and pattern classification, etc. [2], [3]. Neuromorphic processors like IBM's TrueNorth [4], and Intel's Loihi [5] are examples of breakthroughs in the field of neuromorphic engineering that can carry out complex cognitive tasks in the realm of visual and sensory applications. In comparison to the compartmental processing of conventional supercomputers which require MWs of power, neuromorphic-based systems have proved to be successful in achieving the key ingredients of power efficiency (orders of less magnitude), accuracy and superior performance in mimicking the human brain [6]. Therefore, research interests in neuromorphic engineering has

Jani Babu Shaik, Sonal Singhal is with the Department of Electrical Engineering, Shiv Nadar University, UP,201314, India (e-mail: *skjanibabu786@gmail.com and sonal.singhal@snu.edu.in). risen in recent decades towards becoming the current prime contender for progress in futuristic technology.

Extensive research in the field of neuromorphic engineering in recent years has led to the development of various bioinspired circuits on silicon chips using advanced CMOS technology nodes [6]-[13]. Therefore, it is notable to consider reliability issues such as Bias Temperature Instability (BTI) and Hot Carrier Injection (HCI) [14]-[17] at these advanced nodes. BTI leads to the shift of device parameters like the threshold voltage $\left(\Delta \mathrm{V}_{\mathrm{TH}}\right)$, drain current $\left(\mathrm{I}_{\mathrm{D}}\right)$, subthreshold slope $(\Delta \mathrm{S})$, transconductance $(\mathrm{gm})$ because of gradual accumulation of charges at or near gate insulator [18]-[24]. HCI affects similar device parameters as BTI with charge carriers in the channel attaining high energy due to a rise in the electric field [25]-[29]. In addition to reliability issues, process (time-zero) variabilities such as random dopant fluctuation (RDF), Metal Gate Granularity (MGG), and line edge roughness (LER) deviate device parameters from its mean value [30]-[39] and eventually affect the circuit performance [41, 42].

Reliability analysis aids to estimate the impact of aging issues on system performance over its lifetime providing a quantitative assessment at the design stage empowering designers to set guard band or design margins before the manufacturing process [42].

Reliability analysis has been predominantly carried out on logic circuits such as flip-flops, SRAMs, logic gates, etc. due to its feature size scaling and thus set design guard bands [43][51]. Additionally, limited reliability research has been focused on analog/mixed VLSI circuits such as transconductance amplifiers, digital to analog (DAC) circuits, etc. [20], [52][61][61]. SiN circuits falls in the category of analog/mixed VLSI circuits and their performance is susceptible to degradation due to both BTI and HCI degradation mechanisms. Degradation in neuron circuit may result in the neural network failure. This motivates us to explore neuromorphic circuits from a reliability point of view and propose reliability aware SiN designs that can provide a solution to sustain device degradation. To the best of our knowledge, this is the first study addressing reliability concerns of spiking-events in neuromorphic circuits along with a novel approach employed to enhance the designs from its degradation perspective.

This work is focused on two neuromorphic circuits based on the I\&F model; the popular axon-hillock (AH) and simplified leaky integrate-and-fire (SLIF) circuits that has been used in different applications [8], [9], [62]-[68]. These circuits are implemented in the lower frequency ranges from $65 \mathrm{~Hz}$ to

Siona Menezes Picardo, Nilesh Goel is with the Department of Electrical and Electronics Engineering, BITS Pilani Dubai Campus, Dubai, UAE. (Email: sionacmenezes@gmail.com, goel.nilesh@ gmail.com) 
$1 \mathrm{KHz}$, enabling them to fit into the operational range of biological neurons. Combined impact of BTI and HCI along with the time-zero variability on these neuromorphic circuits are studied in detail.

Sections 2, 3, 4 and 5 of the paper are organized as follows: In section 2, the simulation framework of this work is discussed. Section 3 describes the working of AH and SLIF neuron circuits with their detailed analysis from reliability point of view. In section 4, we present the proposed reliability-aware designs followed by simulation results of the two neuron circuits. Finally, we conclude in section 5.

\section{SIMULATION FRAMEWORK}

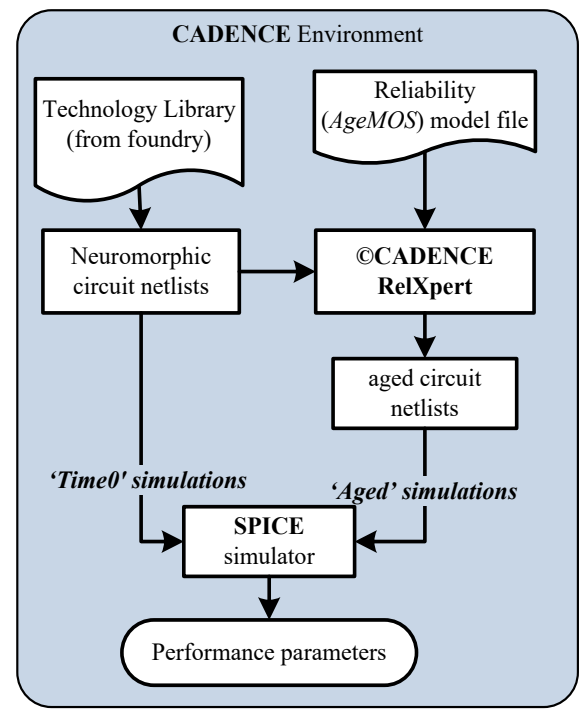

Fig. 1. Simulation framework to evaluate performance of neuromorphic circuit

Fig. 1 shows simulation framework to evaluate the impact of BTI, HCI, and time-zero variability. All simulations are performed in CADENCE environment. Industry-standard 45nm High-K metal gate MOS technology library from foundry is used to create neuromorphic circuits considered for this study. The AgeMOS reliability model [55], [69] provided by the foundry based on BERT (Berkeley Reliability Tool) [70] model is used. Cadence integrates the AgeMOS model in its reliability simulation tool 'Cadence RelXpert'. The reliability tool considers the circuit netlist and voltage applied to the devices through the transient simulation. It then estimates degradation in device parameters after ten years of a lifetime based on the device operating conditions. Circuit netlists and reliability model (AgeMOS) files are fed to the RelXpert tool for generating aged netlists. Time-zero and aged netlists are provided to the SPICE simulator for simulations. Time-zero and aged simulations are referred as 'Time0' and 'Aged' respectively. Aged simulations are performed for combined effects of BTI and HCI; by considering lifetime spans of ten years. Frequency of spiking-events $\left(f_{\mathrm{SPK}}\right)$, spike-ON duration $\left(\mathrm{T}_{\mathrm{ON}}\right)$, spike-OFF duration $\left(\mathrm{T}_{\mathrm{OFF}}\right)$, average power $\left(\mathrm{P}_{\mathrm{avg}}\right)$ of $\mathrm{SiN}$ are considered as the key parameters to analyze the performance of neuron circuits. Performance of aged neuron circuits is characterized by calculating the percentage change in Aged parameter with respect to Time 0 value and is calculated by using equation (1):

$$
\text { Percentage change }=\frac{(\text { Aged }- \text { Time } 0)}{\text { Time } 0} * 100
$$

where Time0 and Aged are performance parameter values of pre- and post-degradations respectively.

For time-zero process variability analysis, 1000 MonteCarlo (MC) simulations are performed. MC simulations are carried out for both AH and SLIF circuits at a constant spiking frequency of $200 \mathrm{~Hz}$.

\section{NEUROMORPHIC CIRCUITS}

This section discusses the operation of two neuromorphic circuits, axon-hillock (AH) and simplified leaky integrate-andfire (SLIF). Impact of BTI and HCI on these neuromorphic circuits are also presented.

\section{A. Axon-Hillock circuit}

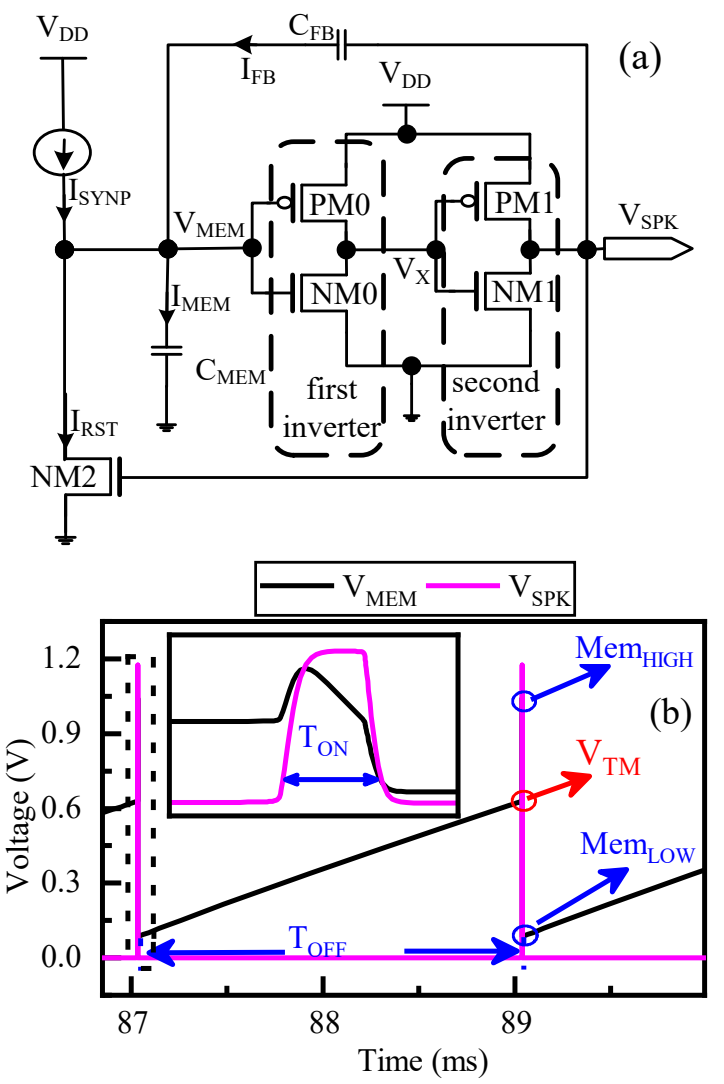

Fig. 2. (a) Schematic of Axon-Hillock (AH) circuit. (b) Simulated waveform of $\mathrm{V}_{\mathrm{MEM}}$ and $\mathrm{V}_{\mathrm{SPK}}$ with performance parameters; zoomed portion of $\mathrm{V}_{\mathrm{MEM}}$ and $\mathrm{V}_{\mathrm{SPK}}$ for spike-ON duration (inset).

The spiking neuron circuit namely the axon-hillock [8], [71] is shown in Fig. 2(a). An external current source ( $\left.\mathrm{I}_{\mathrm{SYNP}}\right)$ models the synaptic current which charges the membrane capacitor $\left(\mathrm{C}_{\mathrm{MEM}}\right)$ and thus develops a membrane voltage ( $\left.\mathrm{V}_{\mathrm{MEM}}\right)$. Membrane voltage increases until it reaches the membranethreshold $\left(\mathrm{V}_{\mathrm{TM}}\right)$. $\mathrm{V}_{\mathrm{TM}}$ is governed by switching point of the first inverter (PM0-NM0) which raises the $\mathrm{V}_{\mathrm{SPK}}$ to $\mathrm{V}_{\mathrm{DD}}$. It provides positive feedback to $\mathrm{V}_{\mathrm{MEM}}$ through $\mathrm{PM} 1$ and $\mathrm{C}_{\mathrm{FB}}$, and causes a steep charging of $\mathrm{C}_{\mathrm{MEM}}$. Simultaneously negative feedback path is activated and reset current (I $\mathrm{I}_{\mathrm{RST}}$ ) flows through NM2 resulting in slow discharge of $\mathrm{V}_{\text {MEM }}$.

The membrane potential ( $\mathrm{V}_{\text {MEM }}$ ) and generated spike event $\left(\mathrm{V}_{\mathrm{SPK}}\right)$ are shown in Fig. 2(b). Generated spike train 
performance is characterized by parameters such as spike-ON duration $\left(\mathrm{T}_{\mathrm{ON}}\right)$, spike-OFF duration $\left(\mathrm{T}_{\mathrm{OFF}}\right)$, minimum and maximum value of membrane voltage $\mathrm{V}_{\mathrm{MEM}}\left(\mathrm{MEM}_{\mathrm{LOW}}\right.$, and MEM $_{\text {HIGH) }}$. Parameters are marked on $\mathrm{V}_{\mathrm{MEM}}$ waveform as shown in Fig. 2(b). Inset shows zoomed portion of $V_{\text {MEM }}$ and $\mathrm{V}_{\text {SPK }}$ with $\mathrm{T}_{\mathrm{ON}}$ marked. $\mathrm{T}_{\mathrm{ON}}$ and $\mathrm{T}_{\mathrm{OFF}}$ parameters are crucial to analyze the aging behaviour of neuron circuits. Any change in these parameters can cause variation in spike frequency

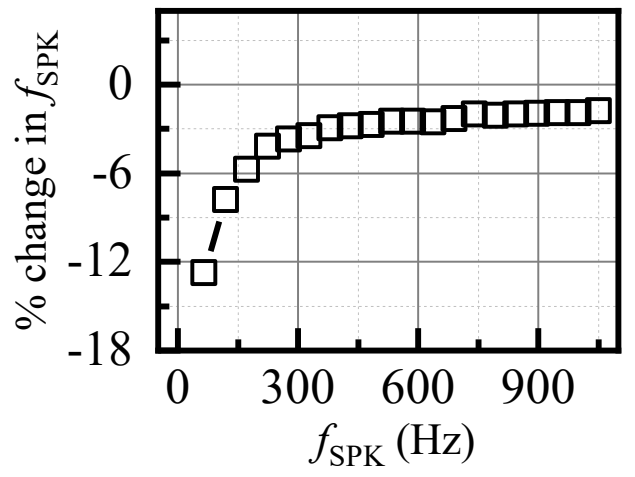

Fig. 3. Percentage change in $f_{\mathrm{SPK}}$ with variation in $f_{\mathrm{SPK}}$ for AH-circuit

Variation in spike frequency due to BTI and HCI degradation is shown in Fig. 3. It is plotted as a percentage change in spike frequency $\left(f_{\mathrm{SPK}}\right)$ with spike frequency $f_{\mathrm{SPK}}$, at the end-of-life performance (after 10 years of circuit operation) [29], [55], [72]-[74]. Percentage change in $f_{\mathrm{SPK}}$ has a larger deviation towards lower frequencies with a maximum value of $13 \%$ as shown in Fig. 3.

Degradation analysis shows that transistors PM0 and NM0 are mainly responsible for performance degradation in $\mathrm{AH}$ circuit. Transistor PM0 suffers from BTI as it remains in inversion mode during $\mathrm{V}_{\mathrm{MEM}}$ integration time. i.e., for $\mathrm{T}_{\mathrm{OFF}}$ time duration. Transistor NM0 degrades due to $\mathrm{HCI}$ as drain current flows during the $\mathrm{T}_{\mathrm{OFF}}$ time. Degradation in transistor PM0 and NM0 affect switching point of the first inverter. It consequently causes a shift in membrane-threshold $\left(\mathrm{V}_{\mathrm{TM}}\right)$ and results in deviation in $f_{\mathrm{SPK}}$ values after degradation. Moreover, shift in $\mathrm{V}_{\mathrm{TM}}$ is similar for all $f_{\mathrm{SPK}}$ values. However, time to charge $\mathrm{C}_{\mathrm{MEM}}$ and eventually to reach to shifted $\mathrm{V}_{\mathrm{TM}}\left(\Delta \mathrm{T}_{\mathrm{OFF}}\right)$ decays exponentially with increase in synaptic current. Thus, percentage deviation in $f_{\text {SPK }}$ shows an increased degradation at low $f_{\mathrm{SPK}}$ values and saturate for high $f_{\mathrm{SPK}}$ values as shown in Fig. 3.

\section{B. Simplified Leaky integrate-and-fire circuit}

Fig. 4(a) shows simplified leaky integrate-and-fire (SLIF) spiking neuron circuit [65]. In SLIF circuit, spiking parameters such as membrane-threshold $\left(\mathrm{V}_{\mathrm{TM}}\right)$, refractory period $\left(\mathrm{T}_{\mathrm{RFR}}\right)$ is governed by external voltage sources. Current source ( $\left.\mathrm{I}_{\mathrm{SYNP}}\right)$ models the synaptic current that charges $\mathrm{C}_{\mathrm{MEM}}$, thus membrane potential $\left(\mathrm{V}_{\mathrm{MEM}}\right)$ develops. Source follower (NM2 \& NM3) with voltage source $\left(\mathrm{V}_{\mathrm{THR}}\right)$ sets the membrane-threshold $\left(\mathrm{V}_{\mathrm{TM}}\right)$. Output of source follower $\left(\mathrm{V}_{\mathrm{SF}}\right)$ is provided as input to the first inverter (PM2-NM4). Inverter output $\left(\mathrm{V}_{\mathrm{FB}}\right)$ goes low when $\mathrm{V}_{\mathrm{SF}}$ reaches its switching point, leading to a positive feedback current $\left(\mathrm{I}_{\mathrm{FB}}\right)$ through transistor PM1. Consequently, output voltage of the second inverter (PM3-NM5) goes high, allowing a reset current $\left(\mathrm{I}_{\mathrm{RST}}\right)$ to flow through NM1, discharging the
$\mathrm{V}_{\mathrm{MEM}}$ to a resting potential. The refractory period $\left(\mathrm{T}_{\mathrm{RFR}}\right)$ is controlled by transistor NM6 (kept in the sub-threshold region) with voltage source $\left(\mathrm{V}_{\mathrm{RFR}}\right)$. The membrane potential $\left(\mathrm{V}_{\mathrm{MEM}}\right)$ and a generated spike event are shown in Fig. 4(b). Spike train performance is characterized by parameters such as spike-ON duration $\left(\mathrm{T}_{\mathrm{ON}}\right)$ and spike-OFF duration $\left(\mathrm{T}_{\mathrm{OFF}}\right)$. Parameters are marked on $\mathrm{V}_{\text {MEM }}$ waveform as shown in Fig. 4(b). Inset shows zoomed portion of $\mathrm{V}_{\mathrm{MEM}}$ and $\mathrm{V}_{\mathrm{SPK}}$.

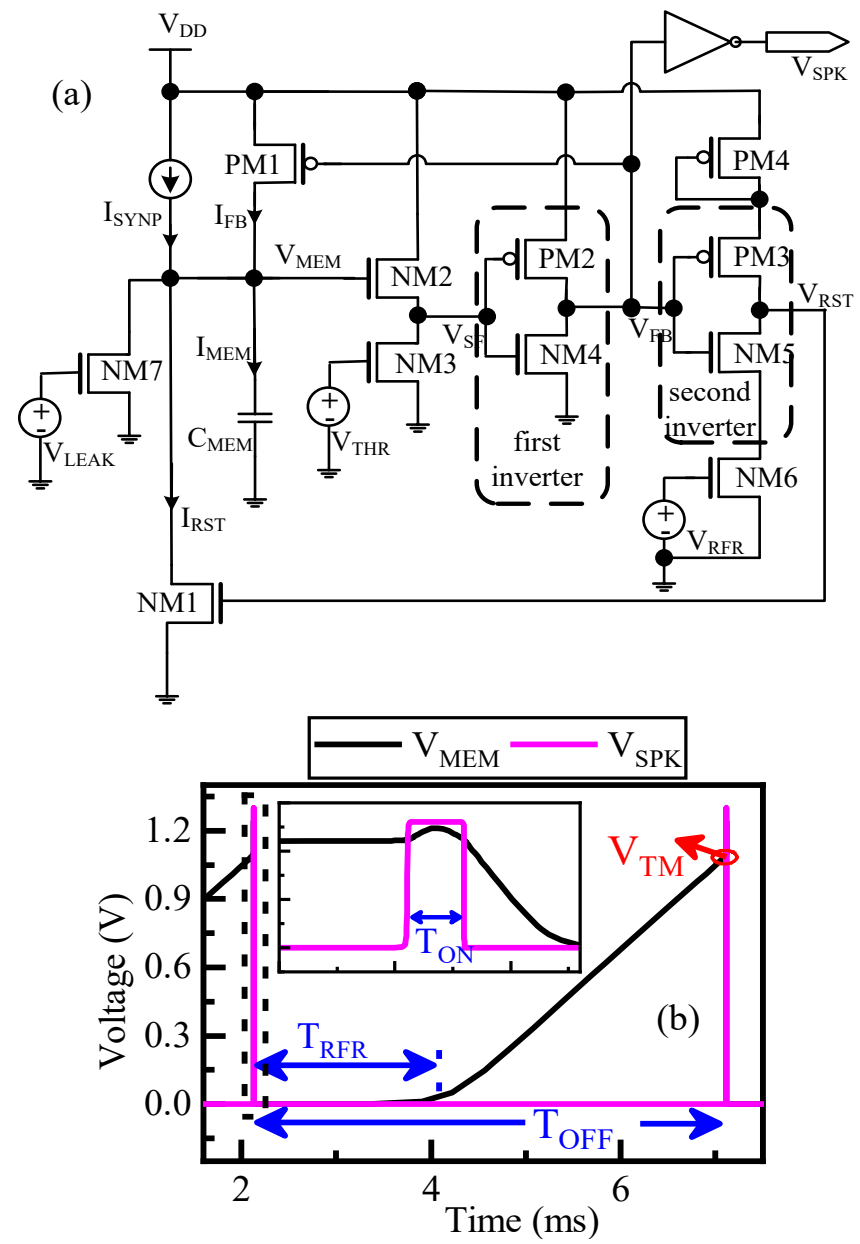

Fig. 4(a) Schematic of simplified leaky integrate-and-fire (SLIF) circuit (b) Simulated waveform of $\mathrm{V}_{\mathrm{MEM}}$ and $\mathrm{V}_{\mathrm{SPK}}$ with performance parameters and refractory period $\left(\mathrm{T}_{\mathrm{RFR}}\right)$; zoomed portion of $\mathrm{V}_{\mathrm{MEM}}$ and $\mathrm{V}_{\mathrm{SPK}}$ for spike-ON duration (inset)

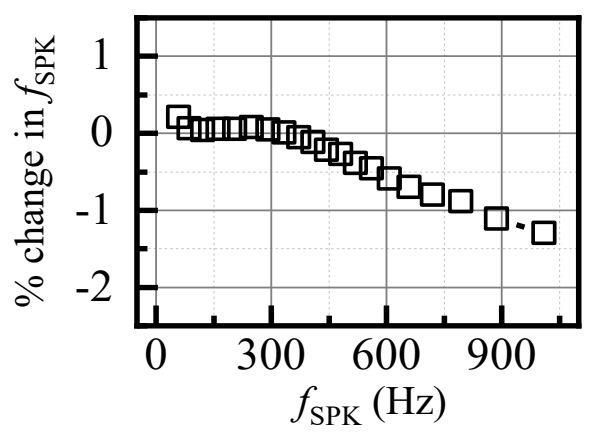

Fig. 5. Percentage change in $f_{\mathrm{SPK}}$ with variation in $f_{\mathrm{SPK}}$ for SLIF circuit

Fig. 5 shows percentage change in $f_{\mathrm{SPK}}$ with variation in spike frequency of SLIF circuit. It is observed that variation in $f_{\mathrm{SPK}}$ 

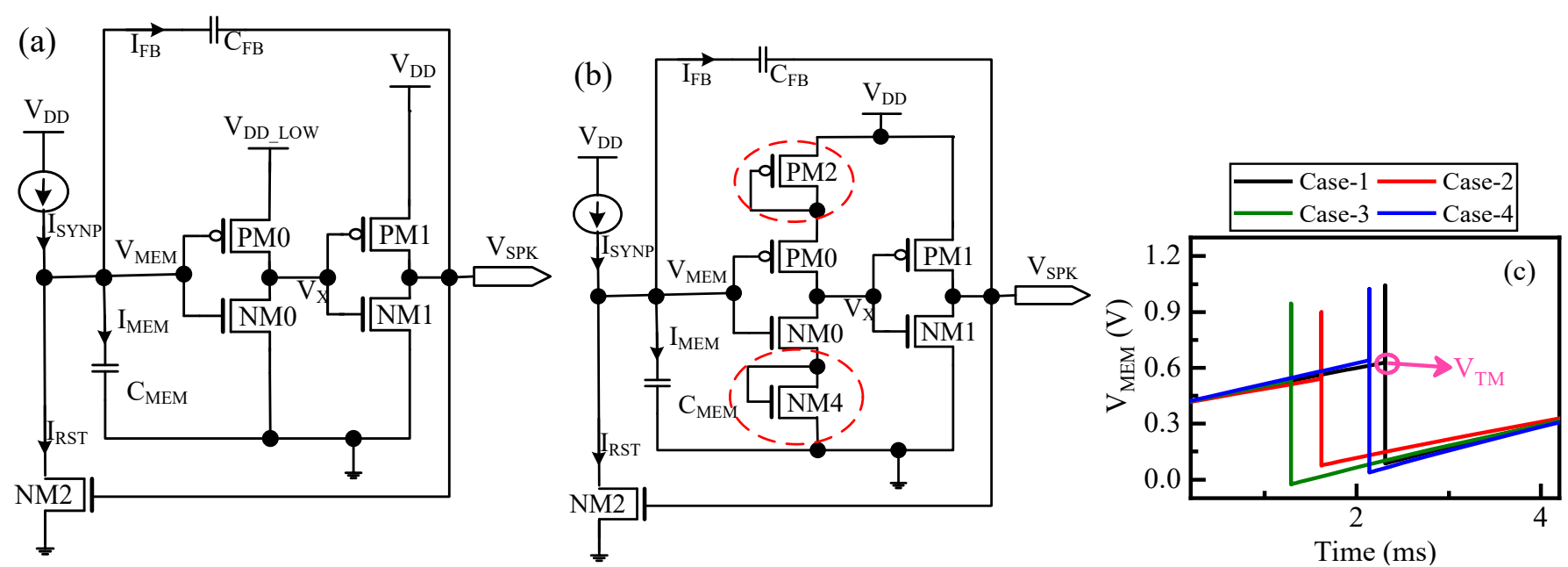

Fig. 6. (a) Schematic of Axon-Hillock circuit with two $V_{D D}$ supply voltages for case-1, case-2 and case-3. (b) Schematic of AH circuit with two diode-connected loads as case-4. (c) Simulated $V_{\text {MEM }}$ for all considered cases

increases marginally for higher values of spike frequency. Degradation analysis shows that transistor PM2 dominantly affects the SLIF neuron circuit performance. Degraded transistor PM2 is responsible for slower discharge of reset voltage $\left(\mathrm{V}_{\mathrm{RST}}\right)$. This results in increased spike-OFF duration $\left(\mathrm{T}_{\mathrm{OFF}}\right)$ and reduced spike frequency $\left(f_{\mathrm{SPK}}\right)$ values after degradation. With increase in $f_{\mathrm{SPK}}$, the degradation of PM2 is also observed to increase. Thus, deviation in $f_{\mathrm{SPK}}$ increases with increase in $f_{\mathrm{SPK}}$ as shown in the figure.

\section{Proposed Reliability-aware NeUromorphic CIRCUITS}

This section discusses the proposed reliability-aware design of axon-hillock (AH) and simplified leaky integrate-and-fire (SLIF) neuron circuits.

TABLE I

LIST OF CONSIDERED CASES FOR MITIGATING RELIABILITY ISSUES IN AXONHILLOCK CIRCUIT

\begin{tabular}{ccccc}
\hline \hline Considered cases & Case-1 & Case-2 & Case-3 & Case-4 \\
\hline$V_{D D}$ & 1.3 & 1.1 & 1.3 & 1.3 \\
$V_{D D \_}$LOW & 1.3 & 1.1 & 1.1 & - \\
\hline \hline
\end{tabular}

To minimize the impact of aging induced degradation, techniques like $\mathrm{V}_{\mathrm{DD}}$ lowering can be used; which is commonly proposed in the literature [43], [44], [46], [75]-[77]. It is discussed in section III, that transistors PM0 and NM0 are mainly responsible for performance degradation in $\mathrm{AH}$ neuron circuit. Overdrive voltages of these two transistors can be reduced to mitigate the reliability issues in axon-hillock circuit. Table-I lists the cases which are considered in this work to reduce the impact of aging and process variation induced degradation in AH circuit. Case-1 is the reference case, discussed in section-2, now referred as Conv-AH. In case-2, a global $\mathrm{V}_{\mathrm{DD}}$ scaling approach is resorted to as a means to reduce $\mathrm{V}_{\mathrm{DD}}$ in all transistors. In case-3, a selective $\mathrm{V}_{\mathrm{DD}}$ scaling approach is used by employing two distinguished power levels

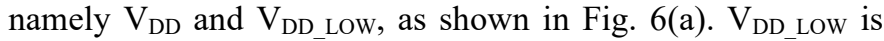
connected to transistors namely NM0 and PM0, while rest of the circuit operates on $V_{D D}$. Case-4 is our proposed reliabilityaware AH (Rel-AH) circuit consists of diode connected transistors to reduce overdrive voltages of NM0 and PM0 transistors as shown in Fig. 6(b). Adding these additional transistors increases the area overhead of AH neuron circuit by $\sim 15 \%$. Table-I also lists the supply voltage values used in simulations. Fig. 6(c) shows $\mathrm{V}_{\text {MEM }}$ for each case. It is observed from Fig. 6(c) that membrane potential waveform remains intact for all cases and only a shift in membrane-threshold $\left(\mathrm{V}_{\mathrm{TM}}\right)$ is observed with respect to Conv-AH.

Fig. 7 shows the percentage change in $\mathrm{V}_{\mathrm{TH}}$ of degraded transistors for all considered cases of AH circuit. The shift in $\mathrm{V}_{\mathrm{TH}}$ of NM0 and PM0 for case-2, case- 3 and case- 4 are found to have lower values with respect to case-1 (Conv-AH). For case- 2 and case- $3, V_{\mathrm{DD}}$ lowering effects in reducing stress on the transistors which causes a lesser drain current in the first inverter. This results in significant drop of $\mathrm{V}_{\mathrm{TH}}$ shift with reference case. For case-4, the additional transistors reduce drain to source voltages of both NM0 and PM0 and eventually lower the drain current in that path. This leads to very negligible shift in $\mathrm{V}_{\mathrm{TH}}(\sim 0.5 \%)$ of NM0 and is shown in Fig. 7.

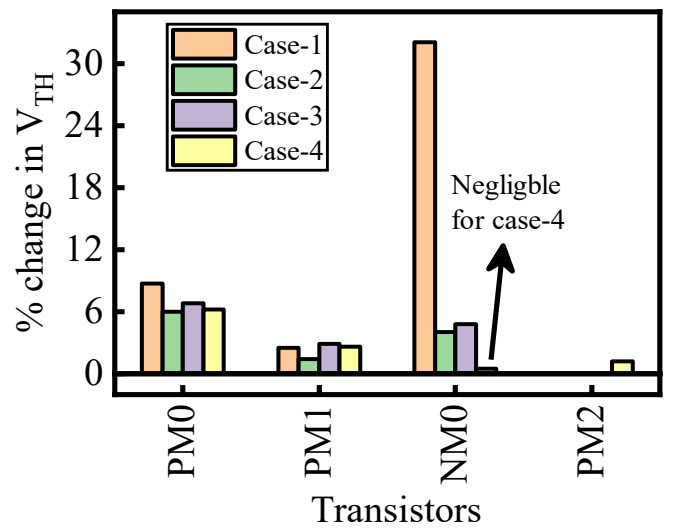

Fig. 7. Percentage change in $\mathrm{V}_{\mathrm{TH}}$ of degraded transistors for all considered cases of AH circuits. Figure shows transistors whose degradation values are greater than $1 \%$.

Fig. 8 shows the percentage change in $f_{\mathrm{SPK}}$ with spike frequency. Case-1 shows significant deviation $(\sim 13 \%)$ for low 
$f_{\mathrm{SPK}}$ values and gets saturated for higher $f_{\mathrm{SPK}}$ values. This behavior of percentage change in $f_{\mathrm{SPK}}$ with spike frequency is discussed earlier in section-II. All other cases show less than $3 \%$ deviation for full range of $f_{\mathrm{SPK}}$ values. This is attributed to the reduced overdrive voltages of transistors NM0 and PM0. Monte-Carlo (MC) simulations are performed, at $f_{\mathrm{SPK}}$ of $200 \mathrm{~Hz}$, to analyze time-zero process variability. Fig. 9 shows probit plots of $f_{\mathrm{SPK}}$ for all cases. Probit plots shows that $f_{\mathrm{SPK}}$ in each case follows the Gaussian distribution. Corresponding standard deviation (SD) is shown in figure. Case-4 shows the least standard deviation amongst the considered cases. Case-4 (Rel$\mathrm{AH}$ ) is therefore a superior circuit with equivalent mitigation of reliability degradation projecting least impact of process variability. Hence from here we will compare the performance of Rel-AH with Conv-AH neuron circuits.

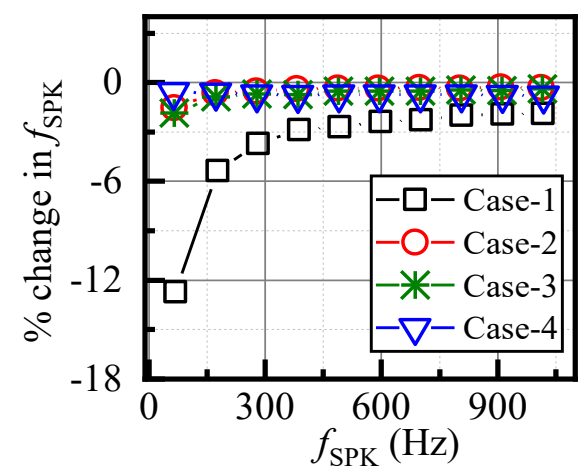

Fig. 8. Percentage change in $f_{\mathrm{SPK}}$ with variation in $f_{\mathrm{SPK}}$ for considered cases of AH circuit.

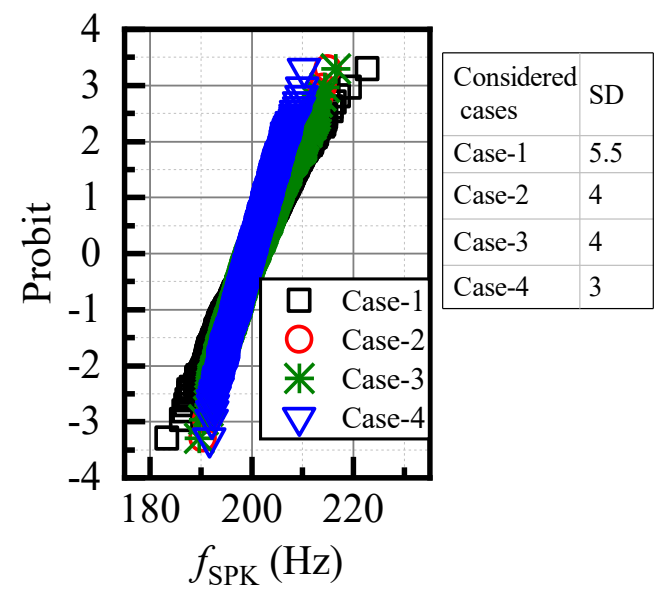

Fig. 9. Probit plot spike frequency $\left(f_{\mathrm{SPK}}\right)$ for considered of AH circuit. Standard deviation (SD) values of each considered case is shown in the table.

Fig. 10 shows the normalized average power dissipation of the AH neuron circuit for all cases at time-zero. The average power is normalized to the power dissipation value obtained at the lowest frequency point in the reference case (case-1). The power dissipation in the $\mathrm{AH}$ circuit is mainly due to the first inverter, which is $\mathrm{ON}$ for the entire spike-OFF duration $\left(\mathrm{T}_{\mathrm{OFF}}\right)$. Case- 2 and Case- 3 both employ the $\mathrm{V}_{\mathrm{DD}}$ scaling, thereby reducing power dissipation w.r.t case-1, also evident from Fig. 10. Proposed reliability-aware design (case-4) shows even lesser power dissipation. Case-4 employs the diode-connected transistors (PM2 and NM4), which reduces the gate-source voltage of transistors NM0 and PM0. The decreased gatesource voltages of NM0 and PM0 result in the lesser current drive of the first inverter, reducing overall power dissipation in case-4.

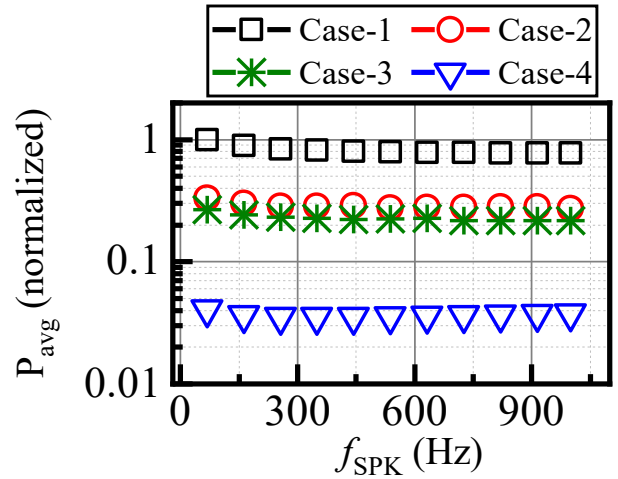

Fig. 10. Normalized average power dissipation with variation in $f_{\mathrm{SPK}}$ for all considered cases of $\mathrm{AH}$ neuron circuits at time-zero.

Impact of BTI \& HCI degradation on membrane voltage is characterized by measuring the difference in Time 0 membrane voltage and Aged membrane voltage. It is expressed as the difference in $M E M_{L O W}$ or $M E M_{H I G H}$ for membrane high and low potential respectively at time-zero and aged circuits. Fig. 11(a) and (b) shows difference in $M E M_{L O W}$ and $M E M_{H I G H}$ for Conv$\mathrm{AH}$ and Rel-AH circuits. Conv-AH circuit shows difference of $\sim 60 \mathrm{mV}$ in $\mathrm{MEM}_{\text {LOw }}$ and $\mathrm{MEM}_{\text {HIGH }}$ due to degradation. It is attributed to the degradation in NM0 and PM0 transistors. RelAH circuit shows significant reduction in the difference in MEM $_{\text {LOW }}$ and MEM $_{\text {HIGH }}$.

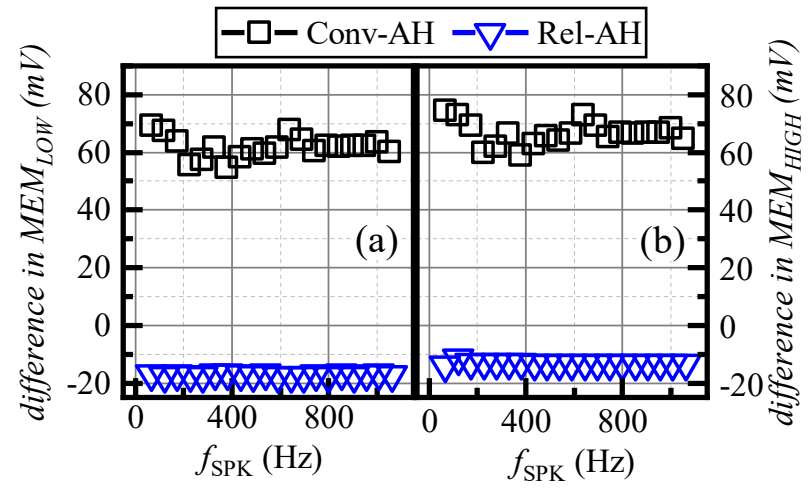

Fig. 11. Voltage difference of Aged (a) $\mathrm{MEM}_{\mathrm{LOW}}$ and (b) $\mathrm{MEM}_{\mathrm{HIGH}}$ respect to Time 0 with variation in $f_{\mathrm{SPK}}$ for Conv-AH and Rel-AH circuit

Fig. 12(a) and (b) shows percentage change in spike-ON duration ( $\mathrm{T}_{\mathrm{ON}}$ ) and spike-OFF duration ( $\left.\mathrm{T}_{\mathrm{OFF}}\right)$ with $f_{\mathrm{SPK}}$ respectively. Both Conv-AH and Rel-AH circuits show negligible deviation $(\sim 1.5 \%)$ in $\mathrm{T}_{\mathrm{ON}}$ with $f_{\mathrm{SPK}}$. The Conv-AH circuit shows a significant higher percentage change $(\sim 15 \%)$ in $\mathrm{T}_{\mathrm{OFF}}$ for lower $f_{\mathrm{SPK}}$ values, while it is very less in the proposed Rel-AH circuit. Each circuit shows negligible percentage change in $\mathrm{T}_{\mathrm{OFF}}$ for higher $f_{\mathrm{SPK}}$ values. 


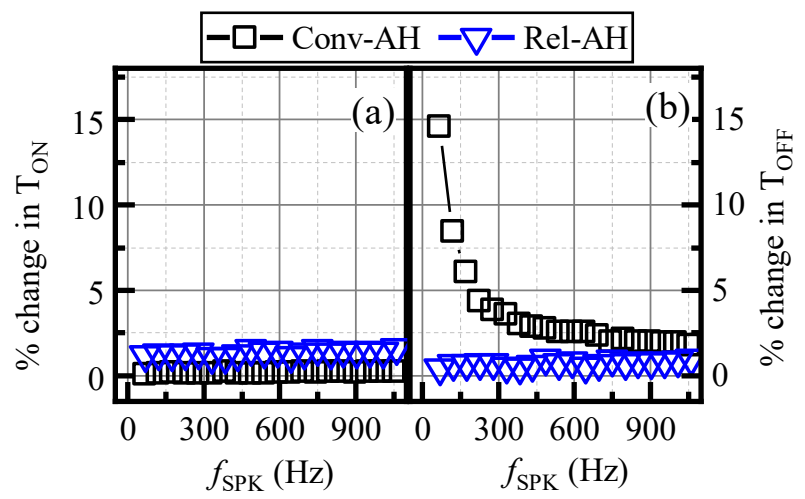

Fig. 12. Percentage change in (a) $\mathrm{T}_{\mathrm{ON}}$ (b) $\mathrm{T}_{\mathrm{OFF}}$ with variation in $f_{\mathrm{SPK}}$ for Conv$\mathrm{AH}$ and Rel-AH circuits

TABLE II

LIST OF CONSIDERED CASES FOR MITIGATING RELIABILITY ISSUES IN SIMPLIFIED LEAKY INTEGRATE-AND-FIRE (SLIF) CIRCUIT

\begin{tabular}{cccc}
\hline \hline Considered cases & Case-1 & Case-2 & Case-3 \\
\hline$V_{D D}$ & 1.3 & 1.1 & 1.3 \\
\hline \hline
\end{tabular}

The performance degradation in SLIF neuron circuit is discussed in section-III. It is largely due to the BTI and HCI degradation in transistor PM2. Table-II list cases considered to mitigate reliability issues in SLIF neuron circuit.
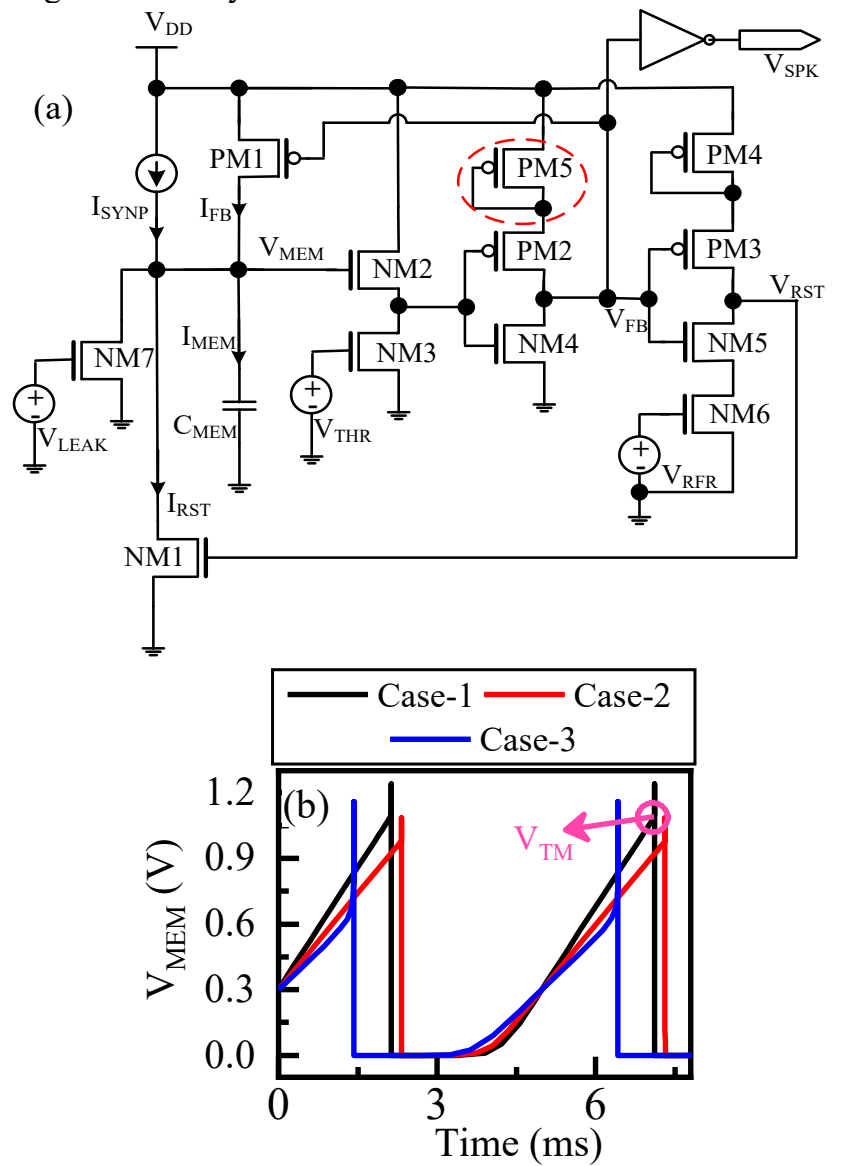

Fig. 13. (a) Schematic of SLIF circuit for case-3 (b) Simulated $V_{M E M}$ for considered three cases

Case-1 is the conventional SLIF neuron and is referred as Conv-SLIF. In case-2, global $\mathrm{V}_{\mathrm{DD}}$ scaling approach is sought.
In case-3, a diode-connected transistor PM5 is used to reduce the overdrive voltage of PM2 to mitigate the reliability issues. Adding transistor PM5 increases the area overhead of SLIF neuron circuit by $\sim 11 \%$. Table also lists supply voltage values used in simulations. SLIF circuit with diode connected transistor PM5 is shown in Fig. 13(a). Fig. 13(b) shows simulated waveforms of membrane voltage for each case. It is observed that membrane voltage shape remains intact while a shift in membrane-threshold $\left(\mathrm{V}_{\mathrm{TM}}\right)$ is observed with respect to Conv-SLIF.

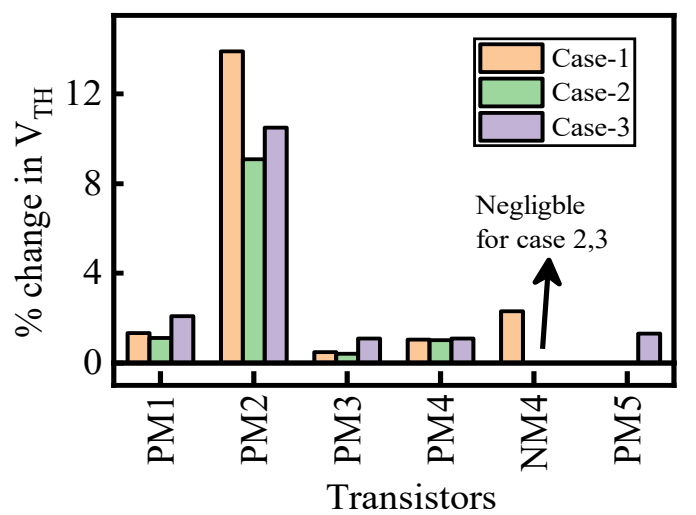

Fig. 14. Percentage change in $\mathrm{V}_{\mathrm{TH}}$ of degraded transistors for all considered cases of SLIF circuits. Figure shows transistors whose degradation values are greater than $1 \%$.

Fig. 14 shows the percentage change in $\mathrm{V}_{\mathrm{TH}}$ of the degraded transistors for all considered cases of SLIF neuron circuit. The shift in $\mathrm{V}_{\mathrm{TH}}\left(\Delta \mathrm{V}_{\mathrm{TH}}\right)$ of PM2 for case-2, case-3 are found to have lower values with respect to case-1 (Conv-SLIF). For case-2, overall $V_{D D}$ scaling effects drain to source voltage of PM2 but shows an increase in $\Delta \mathrm{V}_{\mathrm{TH}}$ of NM4 due to HCI. For case- 3 with the additional transistor (PM5), drain to source voltage of PM2 always has a diode drop which leads in reduced $\mathrm{V}_{\mathrm{TH}}$ shift of PM2 transistor as shown in figure.

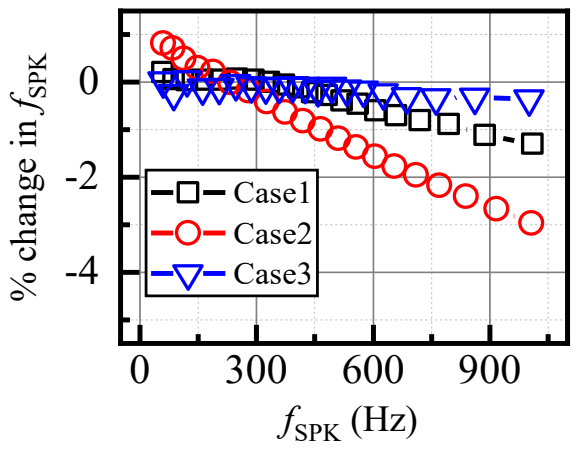

Fig. 15. Percentage change in $f_{\mathrm{SPK}}$ with variation in $f_{\mathrm{SPK}}$ for considered cases SLIF circuits

Fig. 15 shows the percentage change in $f_{\text {SPK }}$ with $f_{\text {SPK }}$. For case- 1 and case-2, percentage deviation increases with spike frequency. Case-3 shows a negligible percentage change in $f_{\mathrm{SPK}}$ for all $f_{\mathrm{SPK}}$ values. Diode-connected transistor reduces drain to source voltage of transistor PM2 and results in lesser degradation value compared to Conv-SLIF neuron circuit, thereby reducing the percentage change in $f_{\text {SPK }}$ with variation in $f_{\text {SPK. }}$. Time-zero variability simulations are performed for considered cases of SLIF neuron circuit. Fig. 16 shows probit 
plots of spike frequency. For all cases, probit plots follow the Gaussian distribution with larger deviation at tail ends. Standard deviation (SD) values for each case are shown in the figure. It is observed that SD of case- 1 and case- 3 are in similar range and are higher than case-2. Case- 2 shows less deviations with process variations but it is more sensitive to aging as the overdrive voltage of the second inverter has been compensated with $\mathrm{V}_{\mathrm{DD}}$ value. This results in the slow charging and discharging of $\mathrm{V}_{\text {RST }}$ after degradation. It is noteworthy that case-3 shows an improvement against aging induced degradation. Case-3 is therefore considered as the proposed reliability-aware SLIF (Rel-SLIF). Hence, from now on we will compare only the performance of Rel-SLIF with ConvSLIF neuron circuits.

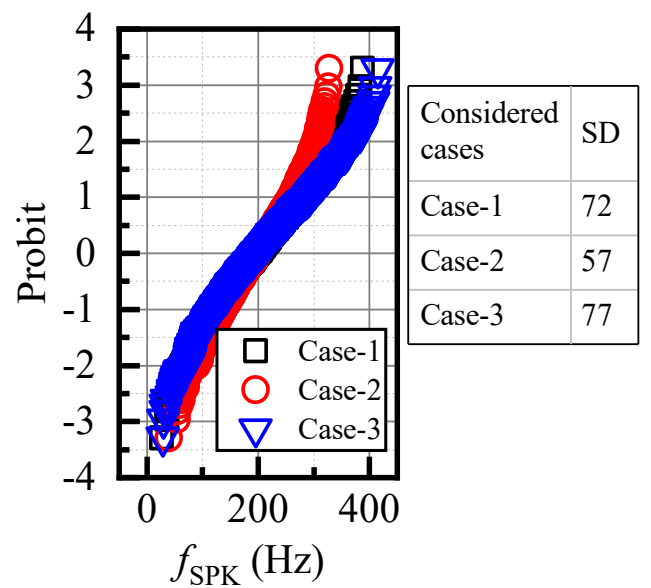

Fig. 16. Probit plot spike frequency $\left(f_{\mathrm{SPK}}\right)$ for considered cases of SLIF circuit. Standard deviation (SD) values of each considered case is shown in the table

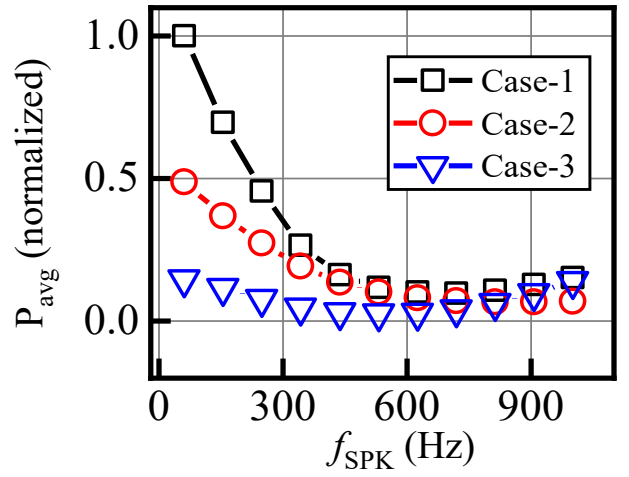

Fig. 17. Normalized average power dissipation for all considered cases of SLIF neuron circuit at time-zero

Fig. 17 shows the normalized average power dissipation of the SLIF neuron circuit for all three cases at time-zero. Power dissipation in the SLIF circuit largely depends on the transistors NM2 and NM3. Both NM2 and NM3 are ON in spike-OFF duration $\left(\mathrm{T}_{\mathrm{OFF}}\right)$. The power dissipation in case-2 is found lesser than case- 1 and is attributed to $V_{D D}$ scaling. In case- 3 , the added transistor PM5 impacts power dissipation in two ways: (i) it reduces the gate-source voltage of transistor NM2 and thus reduces its current (ii) it also reduces membrane-threshold voltage $\left(\mathrm{V}_{\mathrm{TM}}\right)$, as shown in Fig. 13(b). Reduction in $\mathrm{V}_{\mathrm{TM}}$ decreases the time frame for which transistors NM2 and NM3 are ON. Both these effects are coupled together and hence effectively reduce overall power dissipation.

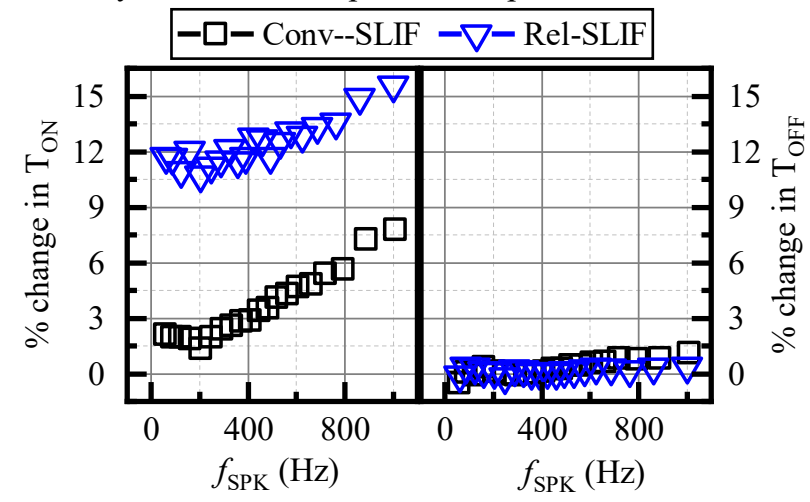

Fig. 18. Percentage change in (a) $\mathrm{T}_{\mathrm{ON}}$ and (b) $\mathrm{T}_{\mathrm{OFF}}$ with variation in $f_{\mathrm{SPK}}$ for Conv-SLIF and Rel-SLIF

Fig. 18(a) shows the percentage change in spike-ON duration $\left(\mathrm{T}_{\mathrm{ON}}\right)$ with variation in $f_{\mathrm{SPK}}$ for Conv-SLIF and Rel-SLIF neuron circuits. For Conv-SLIF neuron circuit, percentage change in $\mathrm{T}_{\mathrm{ON}}$ vary from $1 \%$ to $8 \%$ with $f_{\mathrm{SPK}}$ values. Whereas, proposed Rel-SILF neuron circuit shows a smaller range from $10 \%$ to $15 \%$. Fig. 18 (b) shows percentage change in parameter $\mathrm{T}_{\mathrm{OFF}}$ with $f_{\mathrm{SPK}}$ for Conv-SLIF and Rel-SLIF neuron circuits. Rel-SLIF neuron circuit shows marginal improvement in $\mathrm{T}_{\mathrm{OFF}}$ w.r.t. Conv-SLIF neuron circuit across the frequency range. Although the $\mathrm{T}_{\mathrm{ON}}$ undergoes deviations, the more critical parameter $\mathrm{T}_{\mathrm{OFF}}$ (integration duration of $\mathrm{C}_{\mathrm{MEM}}$ and refractory period) tends to impact the frequency of SLIF neuromorphic circuits, which is least affected. These findings make the proposed reliable-aware circuit effective to mitigate the reliability issues in SLIF circuit.

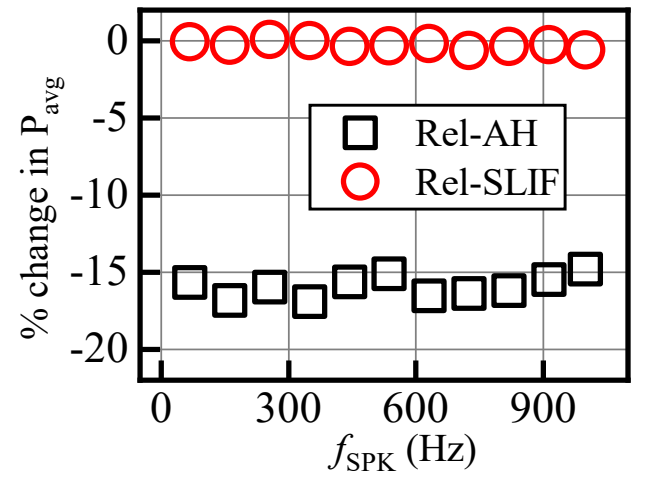

Fig. 19. Percentage change in $\mathrm{P}_{\text {avg }}$ with variation in $f_{\mathrm{SPK}}$ for Rel-AH and RelSLIF.

Fig. 19 shows the percentage change in $\mathrm{P}_{\text {avg }}$ with $f_{\mathrm{SPK}}$ for reliability-aware neuron circuits. Percentage change in average power $\left(\mathrm{P}_{\text {avg }}\right)$ is calculated by considering power dissipation at time-zero and aged reliability-aware neuron circuits. The reliability-aware AH neuron circuit shows $\sim 15 \%$ improvement, whereas the SLIF circuit does not show any change.

\section{CONCLUSION}

In this paper, a detailed analysis of reliability (BTI and $\mathrm{HCI}$ ) impact and time-zero variability were carried out on the key performance parameters of two spike-event neuron circuits namely AH and SLIF. Modified reliability-aware AH and SLIF 
circuits are proposed. Conv-AH shows a larger deviation in spike frequency for lower range of $f_{\mathrm{SPK}}$ values while Conv-SLIF circuit projects a marginal $f_{\mathrm{SPK}}$ deviation with increasing $f_{\mathrm{SPK}}$. Reliability analysis aided in identifying the transistors responsible for performance degradation in Conv-AH and Conv-SLIF circuits. In order to mitigate reliability issues, global and local $V_{D D}$ scaling approaches were considered. The proposed reliability-aware design consisting of diodeconnected transistors was found to be most effective in lowering degradation of transistors in both $\mathrm{AH}$ and SLIF circuits. Consequently, percentage change in $f_{\mathrm{SPK}}$ of the proposed reliability-aware AH and SLIF circuits show negligible deviation in all $f_{\mathrm{SPK}}$ values across considered range. Results of the time-zero variability analysis indicates an improvement in standard deviation (SD) of Rel-AH over ConvAH whereas SD of Rel-SLIF and Conv-SLIF were approximately same. Reliability-aware neuron circuits show significantly lesser power dissipation compared to conventional neuron circuits. This work demonstrates the significance of considering the aspect of reliability in neuromorphic circuit designs, enabling development of robust neuromorphic systems.

\section{ACKNOWLEDGMENT}

The authors are grateful to the Electrical Engineering department of Shiv Nadar University for providing simulation tools that are used in this work. The authors would like to thank the Department of Electrical and Electronics Engineering, BITS Pilani - Dubai Campus, UAE, and Cadence Academic Network team for providing essential resources to perform simulations required for the work conducted in this paper.

\section{REFERENCES}

[1] C. D. Schuman et al., "A Survey of Neuromorphic Computing and Neural Networks in Hardware," Oak Ridge, May 2017.

[2] M. Ismail, Neuromorphic Systems Engineering: Neural Networks in Silicon, vol. 447. Boston, MA: Springer US, 1998.

[3] A. Nere, U. Olcese, D. Balduzzi, and G. Tononi, "A Neuromorphic Architecture for Object Recognition and Motion Anticipation Using Burst-STDP," PLoS ONE, vol. 7, no. 5, p. e36958, May 2012.

[4] P. A. Merolla et al., "A million spiking-neuron integrated circuit with a scalable communication network and interface," Science, vol. 345, no. 6197, pp. 668-673, Aug. 2014.

[5] M. Davies et al., "Loihi: A Neuromorphic Manycore Processor with On-Chip Learning," IEEE Micro, vol. 38, no. 1, pp. 82-99, 2018.

[6] G. Indiveri, "Computation in Neuromorphic Analog VLSI Systems," in Neural Nets WIRN Vietri-01. Perspectives in Neural Computing, T. R. and M. M., Eds. Springer, London, 2002, pp. 3-20.

[7] R. Douglas, M. Mahowald, and C. Mead, "Neuromorphic Analogue VLSI," Annual Review of Neuroscience, vol. 18, no. 1, pp. 255-281, Mar. 1995.

[8] G. Indiveri et al., "Neuromorphic Silicon Neuron Circuits," Frontiers in Neuroscience, vol. 5, no. May, pp. 1-23, 2011.

[9] A. Basu, S. Shuo, H. Zhou, M. Hiot Lim, and G. Bin Huang, "Silicon spiking neurons for hardware implementation of extreme learning machines," Neurocomputing, vol. 102, pp. 125-134, 2013.

[10] A. Van Schaik, C. Jin, T. J. Hamilton, S. Mihalas, and E. Niebur, "A log-domain implementation of the Mihalas-Niebur neuron model," Proceedings of 2010 IEEE International Symposium on Circuits and Systems, pp. 4249-4252, 2010.

[11] A. Van Schaik, C. Jin, A. McEwan, and T. J. Hamilton, "A log-domain implementation of the Izhikevich neuron model," in ISCAS 2010 - 2010 IEEE International Symposium on Circuits and Systems: Nano-Bio Circuit Fabrics and Systems, 2010, no. 4, pp. 4253-4256.
[12] D. B. D. Rubin, E. Chicca, and G. Indiveri, "Characterizing the firing properties of an adaptive analog VLSI neuron," Lecture Notes in Computer Science (including subseries Lecture Notes in Artificial Intelligence and Lecture Notes in Bioinformatics), vol. 3141, pp. 189200, 2004

[13] C. Zhao, W. Danesh, B. T. Wysocki, and Y. Yi, "Neuromorphic encoding system design with chaos based CMOS analog neuron," in 2015 IEEE Symposium on Computational Intelligence for Security and Defense Applications (CISDA), 2015, pp. 1-6.

[14] M. H. Woods and B. L. Euzent, "Reliability in MOS integrated circuits," in 1984 International Electron Devices Meeting, 1984, vol. 53, no. 9 , pp. 50-55.

[15] E. Maricau and G. Gielen, Analog IC Reliability in Nanometer CMOS, vol. 53, no. 9. New York, NY: Springer New York, 2013.

[16] A. Kerber and E. A. Cartier, "Reliability Challenges for CMOS Technology Qualifications With Hafnium Oxide/Titanium Nitride Gate Stacks," IEEE Transactions on Device and Materials Reliability, vol. 9, no. 2, pp. 147-162, Jun. 2009.

[17] S. Mahapatra, V. Huard, A. Kerber, V. Reddy, S. Kalpat, and A. Haggag, "Universality of NBTI-From devices to circuits and products," IEEE International Reliability Physics Symposium Proceedings, pp. 5$12,2014$.

[18] B. E. Deal, M. Sklar, A. S. Grove, and E. H. Snow, "Characteristics of the Surface-State Charge (Qss) of Thermally Oxidized Silicon," Journal of The Electrochemical Society, vol. 114, no. 3, p. 266, 1967.

[19] N. K. Jha, P. S. Reddy, D. K. Sharma, and V. R. Rao, "NBTI degradation and its impact for analog circuit reliability," IEEE Transactions on Electron Devices, vol. 52, no. 12, pp. 2609-2615, 2005.

[20] S. Mukhopadhyay, N. Goel, and S. Mahapatra, "A Comparative Study of NBTI and PBTI Using Different Experimental Techniques," IEEE Transactions on Electron Devices, vol. 63, no. 10, pp. 4038-4045, 2016.

[21] D. K. Schroder, "Negative bias temperature instability: What do we understand?," Microelectronics Reliability, vol. 47, no. 6, pp. 841-852, 2007.

[22] M. A. Alam, H. Kufluoglu, D. Varghese, and S. Mahapatra, "A comprehensive model for PMOS NBTI degradation: Recent progress," Microelectronics Reliability, vol. 47, no. 6, pp. 853-862, 2007.

[23] N. Goel, K. Joshi, S. Mukhopadhyay, N. Nanaware, and S. Mahapatra, "A comprehensive modeling framework for gate stack process dependence of DC and AC NBTI in SiON and HKMG p-MOSFETs," Microelectronics Reliability, vol. 54, no. 3, pp. 491-519, 2014.

[24] S. Mahapatra et al., "A comparative study of different physics-based NBTI models," IEEE Transactions on Electron Devices, vol. 60, no. 3, pp. 901-916, 2013.

[25] E. Takeda, N. Suzuki, and T. Hagiwara, "Device performance degradation to hot-carrier injection at energies below the $\mathrm{Si}$ $\mathrm{SiO}<\mathrm{inf}>2</$ inf $>$ energy barrier," in 1983 International Electron Devices Meeting, 1983, pp. 396-399.

[26] Chenming Hu, Simon C. Tam, Fu-Chieh Hsu, Ping-Keung Ko, TungYi Chan, and K. W. Terrill, "Hot-Electron-Induced MOSFET Degradation - Model, Monitor, and Improvement," IEEE Journal of Solid-State Circuits, vol. 20, no. 1, pp. 295-305, Feb. 1985.

[27] C. R. Parthasarathy et al., "Designing in reliability in advanced CMOS technologies," Microelectronics Reliability, vol. 46, no. 9-11, pp. 1464-1471, 2006.

[28] V. Huard, C. Parthasarathy, A. Bravaix, C. Guerin, and E. Pion, "CMOS device design-in reliability approach in advanced nodes," in 2009 IEEE International Reliability Physics Symposium, 2009, pp. 624-633.

[29] T. Grasser, Hot Carrier Degradation in Semiconductor Devices. Cham: Springer International Publishing, 2015.

[30] M. J. M. Pelgrom, A. C. J. Duinmaijer, and A. P. G. Welbers, "Matching Properties of MOS Transistors," IEEE Journal of SolidState Circuits, vol. 24, no. 5, pp. 1433-1439, 1989.

[31] D. J. Frank, Y. Taur, M. Ieong, and H.-S. P. Wong, "Monte Carlo modeling of threshold variation due to dopant fluctuations," in 1999 Symposium on VLSI Technology. Digest of Technical Papers (IEEE Cat. No.99CH36325), 1999, pp. 169-170.

[32] A. Asenov, A. R. Brown, J. H. Davies, S. Kaya, and G. Slavcheva, "Simulation of intrinsic parameter fluctuations in decananometer and nanometer-scale MOSFETs," IEEE Transactions on Electron Devices, vol. 50, no. 9, pp. 1837-1852, 2003.

[33] K. Agarwal and S. Nassif, "Characterizing process variation in 
nanometer CMOS," Proceedings - Design Automation Conference, pp. 396-399, 2007.

[34] T. Tsunomura et al., "Analyses of $5 \sigma$ Vth fluctuation in $65 \mathrm{~nm}$ MOSFETs using Takeuchi plot," Digest of Technical Papers Symposium on VLSI Technology. pp. 156-157, 2008.

[35] W. Zhao et al., "Rigorous extraction of process variations for 65-nm CMOS design," IEEE Transactions on Semiconductor Manufacturing, vol. 22, no. 1, pp. 196-203, 2009.

[36] A. Asenov et al., "Simulation of statistical variability in nano-CMOS transistors using drift-diffusion, Monte Carlo and non-equilibrium Green's function techniques," Journal of Computational Electronics, vol. 8, no. 3-4, pp. 349-373, 2009.

[37] A. Asenov, "Simulation of statistical variability in nano MOSFETs," Digest of Technical Papers - Symposium on VLSI Technology, vol. 1, no. 2006, pp. 86-87, 2007.

[38] G. Indalecio, A. Garcia-Loureiro, M. Aldegunde, and K. Kalna, "Study of statistical variability in nanoscale transistors introduced by LER, RDF and MGG," Proceedings of the 2013 Spanish Conference on Electron Devices, CDE 2013, pp. 95-98, 2013.

[39] S. S. Sapatnekar, "Overcoming variations in nanometer-scale technologies," IEEE Journal on Emerging and Selected Topics in Circuits and Systems, vol. 1, no. 1, pp. 5-18, 2011.

[40] Y. Ye, S. Gummalla, C. C. Wang, C. Chakrabarti, and Y. Cao, "Random variability modeling and its impact on scaled CMOS circuits," Journal of Computational Electronics, vol. 9, no. 3-4, pp. 108-113, 2010.

[41] J. B. Shaik, S. Singhal, and N. Goel, "Analysis of SRAM metrics for data dependent BTI degradation and process variability," Integration, vol. 72, pp. 148-162, May 2020.

[42] V. Huard, C. R. Parthasarathy, A. Bravaix, T. Hugel, C. Guérin, and E. Vincent, "Design-in-reliability approach for NBTI and hot-carrier degradations in advanced nodes," IEEE Transactions on Device and Materials Reliability, vol. 7, no. 4, pp. 558-570, 2007.

[43] D. Atienza et al., "Reliability-aware design for nanometer-scale devices," in 2008 Asia and South Pacific Design Automation Conference, 2008, pp. 549-554.

[44] M. Alam, "Reliability- and process-variation aware design of integrated circuits," Microelectronics Reliability, vol. 48, no. 8-9, pp. 1114-1122, 2008.

[45] J. Huang, S. Barner, A. Raabe, C. Buckl, and A. Knoll, “A framework for reliability-aware embedded system design on multiprocessor platforms," Microprocessors and Microsystems, vol. 38, no. 6, pp. 539551, Aug. 2014.

[46] H. Amrouch, B. Khaleghi, A. Gerstlauerz, and J. Henkel, "Reliabilityaware design to suppress aging," Proceedings - Design Automation Conference, vol. 05-09-June, pp. 0-5, 2016.

[47] H. Amrouch and J. Henkel, "Containing guardbands," Proceedings of the Asia and South Pacific Design Automation Conference, ASP-DAC, pp. 537-542, 2017.

[48] J. Henkel and H. Amrouch, "Designing reliable, yet energy-efficient guardbands," in 2016 IEEE International Conference on Electronics, Circuits and Systems (ICECS), 2016, pp. 540-543.

[49] R. Huang et al., "Variability- and reliability-aware design for $16 / 14 \mathrm{~nm}$ and beyond technology," Technical Digest - International Electron Devices Meeting, IEDM, vol. 7, pp. 12.4.1-12.4.4, 2018.

[50] S. M. Picardo, J. B. Shaik, S. Sahni, N. Goel, and S. Singhal, "Analyzing the Impact of NBTI and Process Variability on Dynamic SRAM Metrics Under Temperature Variations," in Modelling, Simulation and Intelligent Computing, Lecture Notes in Electrical Engineering 659, N. Goel, S. and Hasan, and V. and Kalaichelvi, Eds. Springer Singapore, 2020, pp. 608-616.

[51] S. J. Babu, S. P. Chaudhari, S. Singhal, and N. Goel, "Analyzing Impact of NBTI and Time-Zero Variability on Dynamic SRAM Metrics," in 2018 15th IEEE India Council International Conference (INDICON), 2018, pp. 1-5.

[52] B. Yan, J. Qin, J. Dai, Q. Fan, and J. B. Bernstein, "Reliability simulation and design consideration of high speed ADC circuits," IEEE International Integrated Reliability Workshop Final Report, pp. 125128,2008

[53] S. More, M. Fulde, F. Chouard, and D. Schmitt-Landsiedel, "Reliability analysis of buffer stage in mixed signal application," Advances in Radio Science, vol. 9, no. 1, pp. 225-230, 2011.

[54] C.-H. Ho, K. A. Jenkins, H. Ainspan, E. Ray, and Peilin Song, "The impact of Hot Carrier Injection (HCI) on Voltage Control Oscillator lifetime prediction," in 2014 IEEE International Reliability Physics
Symposium, 2014, pp. 6B.3.1-6B.3.4.

[55] E. Maricau and G. Gielen, Analog IC Reliability in Nanometer CMOS. New York, NY: Springer New York, 2013.

[56] G. G. E. Gielen, E. Maricau, and P. De Wit, "Designing reliable analog circuits in an unreliable world," Proceedings of the Custom Integrated Circuits Conference, pp. 4-7, 2012.

[57] G. Gielen, E. Maricau, and P. De Wit, "Analog circuit reliability in sub32 nanometer CMOS: Analysis and mitigation," Proceedings -Design, Automation and Test in Europe, DATE, pp. 1474-1479, 2011.

[58] T. Hillebrand, N. Hellwege, M. Taddiken, K. Tscherkaschin, S. Paul, and D. Peters-Drolshagen, "Stochastic LUT-based reliability-aware design method for operation point dependent CMOS circuits," in 2016 MIXDES - 23rd International Conference Mixed Design of Integrated Circuits and Systems, 2016, pp. 363-368.

[59] H. Cai, H. Petit, and J. F. Naviner, "Reliability aware design of low power continuous-time sigma-delta modulator," Microelectronics Reliability, vol. 51, no. 9-11, pp. 1449-1453, 2011.

[60] M. May, M. Alles, and N. Wehn, "A case study in reliability-aware design: A resilient LDPC code decoder," Proceedings -Design, Automation and Test in Europe, DATE, pp. 456-461, 2008.

[61] S. J. Babu et al., "Investigating the Impact of BTI and HCI on LogDomain Based Mihalas-Niebur Neuron Circuit," in Modelling, Simulation and Intelligent Computing, N. Goel, S. and Hasan, and V. and Kalaichelvi, Eds. Springer Singapore, 2020, pp. 528-536.

[62] C. Mead, Analog VLSI and neural systems. Addison-Wesley Longman Publishing Co., Inc., 1989.

[63] E. Yao and A. Basu, "VLSI Extreme Learning Machine: A Design Space Exploration," IEEE Transactions on Very Large Scale Integration (VLSI) Systems, vol. 25, no. 1, pp. 60-74, Jan. 2017.

[64] J. A. M. Olsson and P. Häfliger, "Mismatch reduction with relative reset in integrate-and-fire photo-pixel array," 2008 IEEE-BIOCAS Biomedical Circuits and Systems Conference, BIOCAS 2008, pp. 277 280, 2008.

[65] C. Zhao, W. Danesh, B. T. Wysocki, and Y. Yi, "Neuromorphic encoding system design with chaos based CMOS analog neuron," in 2015 IEEE Symposium on Computational Intelligence for Security and Defense Applications (CISDA), 2015, pp. 1-6.

[66] C. Zhao, B. T. Wysocki, Y. Liu, C. D. Thiem, N. R. Mcdonald, and Y. Yi, "Spike-time-dependent encoding for neuromorphic processors," ACM Journal on Emerging Technologies in Computing Systems, vol. 12, no. 3, 2015.

[67] C. Zhao, Y. Yi, J. Li, X. Fu, and L. Liu, "Interspike-Interval-Based Analog Spike-Time-Dependent Encoder for Neuromorphic Processors," IEEE Transactions on Very Large Scale Integration (VLSI) Systems, vol. 25, no. 8, pp. 2193-2205, Aug. 2017.

[68] C. Zhao, S. Member, B. T. Wysocki, C. D. Thiem, and N. R. Mcdonald, "Design for Neuromorphic Computing Systems," IEEE Transactions on Multi-Scale Computing Systems, vol. 2, no. 4, pp. 265276, 2016.

[69] CADENCE Team, "Reliability Simulation in Integrated Circuit Design," in Proceedings of the International Cadence User Group Conference, 2003.

[70] R. H. Tu et al., "Berkeley reliability tools-BERT," IEEE Transactions on Computer-Aided Design of Integrated Circuits and Systems, vol. 12, no. 10, pp. 1524-1534, 1993.

[71] C. Mead, "Neuromorphic electronic systems," Proceedings of the IEEE, vol. 78, no. 10, pp. 1629-1636, 1990.

[72] Brian Bailey, "Chip Aging Becomes Design Problem," 2018. .

[73] T. Grasser, Bias Temperature Instability for Devices and Circuits. New York, NY: Springer New York, 2014.

[74] S. Mahapatra, Fundamentals of Bias Temperature Instability in MOS Transistors, vol. 52. New Delhi: Springer India, 2016.

[75] V. M. van Santen, H. Amrouch, J. Martin-Martinez, M. Nafria, and J. Henkel, "Designing guardbands for instantaneous aging effects," in Proceedings of the 53rd Annual Design Automation Conference on $D A C$ '16, 2016, vol. 05-09-June, pp. 1-6.

[76] R. Vattikonda, W. Wang, and Y. Cao, "Modeling and minimization of PMOS NBTI effect for robust nanometer design," Proceedings Design Automation Conference, pp. 1047-1052, 2006.

[77] E. Maricau and G. Gielen, "Efficient variability-aware NBTI and hot carrier circuit reliability analysis," IEEE Transactions on ComputerAided Design of Integrated Circuits and Systems, vol. 29, no. 12. pp. 1884-1893, 2010. 